\title{
How can I cope with redeployment?
}

\author{
During the covid-19 pandemic trainees may be asked to work in unfamiliar environments. Abi \\ Rimmer asks experts how doctors can deal with the change
}

\section{Abi Rimmer}

The BMJ

\section{"You're not alone"}

Max Denning, core surgical trainee, says, "You know more than you realise. As we face the realities of covid-19, extraordinary measures are needed to meet the demand on our health system. To meet this challenge, among other measures, trainees are likely to be redeployed to frontline specialties. While every trainee will feel some degree of anxiety; the experience of, and feelings about, being redeployed will vary with specialty and seniority. For junior trainees, rotating into different specialties is the norm. For senior trainees, rotations are less frequent and become increasingly specialised. Even the most sub-specialised doctors can contribute to the general medical care of patients with covid-19. All trainees develop transferable skills over time; how to manage an acute take, work as a team, escalate, refer appropriately, and most importantly how to care for unwell patients.

"While it may seem isolating and daunting to work outside of the usual scope of your practice, away from your familiar team and environment, you should remember that you are not alone. Despite social distancing, self-isolation, and advice against unessential gatherings, we have never been more connected. Under normal conditions, when managing a patient, advice is usually sought from seniors in the same clinical team or from other specialties. It's unconventional to reach out to colleagues that are at home, working at different hospitals, or even in foreign countries. Of course, you must maintain patient confidentiality and dignity, but this is not business as usual. The medical profession has come together and there's a wealth of resources, knowledge, and experience to support you.

"Finally, while it may feel like there's no light at the end of the tunnel, this pandemic will last a finite amount of time. As trainees, we will need to learn new skills and refresh old ones, but when this pandemic comes to an end, we'll know we did everything we could. We will have overcome feelings of anxiety, worked outside our comfort zones, accepted personal risk, and taken extraordinary measures to have overcome an unprecedented challenge."

\section{"Don't hesitate to seek advice"}

Marika Davies, medicolegal adviser for MDA National, says, "This may be a daunting prospect, especially if you've specialised in a particular area for some time. It may also mean working at a lower level than usual, but you will be of invaluable support to colleagues in specialties under the most pressure in the coming months.

"If you're asked to work in an area outside your usual scope of practice you should familiarise yourself with relevant guidance and protocols beforehand, if possible.

"You should apply basic principles of good medical practice: act in the best interests of the patient at all times, work cooperatively with colleagues to provide safe care, and recognise and work within the limits of your competence. You should make clinical decisions on a case by case basis, taking into account your competence and the availability of other options for care.

"The GMC says that doctors should "use their professional judgment to assess risk to deliver safe care informed by any relevant guidance and the values and principles set out in their professional standards.'

"Don't hesitate to seek advice and support from colleagues; good communication will be essential in ensuring your team knows its capabilities and to make sure you have sufficient information and guidance to work in areas in which you are not familiar.

"If concerns are raised about your decisions or actions, the GMC has confirmed it will take into account relevant factors in the environment in which you were working when considering the specific facts of the case."

\section{"Work within your competence"}

Rob Hendry, medical director at Medical Protection Society, says, "If any doctor is asked to perform a duty that they would not normally undertake, regardless of the circumstances they need to assess whether they have the skills and competency to proceed.

"This will include considering what is in the best interests of the patient. If they don't feel it's safe to proceed and that to do so would place the patient at greater risk of harm than not undertaking the duty that has been requested, then they should advise whoever has asked them to do so and explain their concerns.

"It would be wise to record the details of this deliberation in case it becomes necessary to explain the reasoning behind the decision to act or not to act. 
"If a doctor believes that they're being asked to work in a way that's placing patients at risk of harm they should raise their concerns by following the workplace policy and the GMC's guidance, Raising and Acting on Concerns about Patient Safety. ${ }^{1}$ "The government has indicated that there would be 'emergency indemnity' to enable some healthcare professionals to undertake additional or different duties if required. Our assumption is that this would be state backed as it would be NHS contracted work, specifically to support the government's plan to manage covid-19.
"If Medical Protection members have any concerns about the duties they're currently performing to help manage covid-19, they should contact us."

1 General Medical Council. Raising and acting on concerns about patient safety. www.gmcuk.org/ethical-guidance/ethical-guidance-for-doctors/raising-and-acting-on-concerns.

Published by the BMJ Publishing Group Limited. For permission to use (where not already granted under a licence) please go to http://group.bmj.com/group/rights-licensing/ permissions 